\title{
The short international physical activity questionnaire: cross-cultural adaptation, validation and reliability of the Hausa language version in Nigeria
}

Adewale L Oyeyemi ${ }^{i^{*}}$, Adetoyeje Y Oyeyemi ${ }^{1}$, Babatunde O Adegoke ${ }^{2}$, Fatima O Oyetoke ${ }^{1}$, Habeeb N Aliyu ${ }^{1}$, Salamatu U Aliyu ${ }^{1}$ and Adamu A Rufai ${ }^{1}$

\begin{abstract}
Background: Accurate assessment of physical activity is important in determining the risk for chronic diseases such as cardiovascular disease, stroke, type 2 diabetes, cancer and obesity. The absence of culturally relevant measures in indigenous languages could pose challenges to epidemiological studies on physical activity in developing countries. The purpose of this study was to translate and cross-culturally adapt the Short International Physical Activity Questionnaire (IPAQ-SF) to the Hausa language, and to evaluate the validity and reliability of the Hausa version of IPAQ-SF in Nigeria.

Methods: The English IPAQ-SF was translated into the Hausa language, synthesized, back translated, and subsequently subjected to expert committee review and pre-testing. The final product (Hausa IPAQ-SF) was tested in a cross-sectional study for concurrent (correlation with the English version) and construct validity, and test-retest reliability in a sample of 102 apparently healthy adults.

Results: The Hausa IPAQ-SF has good concurrent validity with Spearman correlation coefficients $(\rho)$ ranging from 0.78 for vigorous activity (Min Week ${ }^{-1}$ ) to 0.92 for total physical activity (Metabolic Equivalent of Task [MET]-Min Week $\left.{ }^{-1}\right)$, but poor construct validity, with cardiorespiratory fitness $(\rho=0.21, p=0.01)$ and body mass index $(\rho=$ $0.22, p=0.04$ ) significantly correlated with only moderate activity and sitting time (Min Week ${ }^{-1}$ ), respectively. Reliability was good for vigorous (ICC $=0.73,95 \% \mathrm{C} . \mathrm{I}=0.55-0.84$ ) and total physical activity (ICC $=0.61,95 \%$ C.I $=$ $0.47-0.72)$, but fair for moderate activity (ICC $=0.33,95 \% \mathrm{C.I}=0.12-0.51)$, and few meaningful differences were found in the gender and socioeconomic status specific analyses.
\end{abstract}

Conclusions: The Hausa IPAQ-SF has acceptable concurrent validity and test-retest reliability for vigorous-intensity activity, walking, sitting and total physical activity, but demonstrated only fair construct validity for moderate and sitting activities. The Hausa IPAQ-SF can be used for physical activity measurements in Nigeria, but further construct validity testing with objective measures such as an accelerometer is needed.

\section{Background}

There is a wide consensus on the benefits of physical activity in health and in disease [1]. Accurate assessment of physical activity is important in determining the risk for cardiovascular disease, stroke, type 2 diabetes, cancer and obesity in developing countries [2,3]. One viable

\footnotetext{
* Correspondence: alaoyeyemi@yahoo.com

'Department of Physiotherapy, College of Medical Sciences, University of Maiduguri, Nigeria

Full list of author information is available at the end of the article
}

strategy for stemming the rising incidence of chronic diseases in developing countries is to increase the population's level of physical activity [3,4], but epidemiological studies on physical activity remain sparse in sub-Saharan Africa, due to the lack of culturally relevant physical activity tools in indigenous African languages and an appropriate system for monitoring [5].

The International Physical Activity Questionnaire (IPAQ) was developed as an instrument for cross-national assessment of physical activity and for standardizing

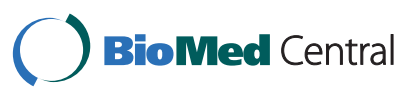


measures of health-related physical activity behaviors of population in many countries and in different sociocultural contexts $[6,7]$. Both the long (31-item) and the short (7-item) versions of this instrument, which elicits information on physical activity over the 'last seven-day' period, have been used to compare physical activity behaviors among and between populations [6,8-10]. The short form of IPAQ (IPAQ-SF) has been recommended for population prevalence studies, where time is limited, because it is easier and more feasible to complete than the long form [6].

The IPAQ has been translated into many languages and extensively tested in many countries around the world, except in sub-Saharan Africa [7,11-15]. In order to make the IPAQ applicable for research among nonEnglish speaking populations in sub-Saharan Africa, it needs to be translated, culturally adapted and properly evaluated for psychometric properties. In sub-Saharan Africa where the sociocultural and physical environments are distinct from those of other parts of the world [16], mere translation of the IPAQ may be insufficient to maintain content validity, as important cultural differences can produce a translated version that lacks experiential and conceptual equivalence [17].

Culturally adapting the IPAQ, rather than developing a new physical activity questionnaire, is economical and it may facilitate future comparisons among populations. In West Africa, Hausa is a widely spoken language with over 50 million native speakers and 15 million non-native speakers in Northern Nigeria, the Republic of Niger, Northern Cameroon and Ghana [18]. The purpose of this study was to translate and cross-culturally adapt the English IPAQ-SF, and to evaluate aspects of the validity and reliability of the Hausa-translated and culturally adapted version of the IPAQ-SF.

\section{Methods}

\section{Setting}

Maiduguri is the largest city and the capital city in the state of Borno. This state is located in North-Eastern Nigeria, and has an estimated population of 4, 151, 193 people, consisting of Kanuri, Shuwa Arabs, Hausa, Fulfude and other ethnic groups $[19,20]$. The state covers an area of 72, 609 sq kilometer with a population density of 57 people/square kilometer, and attracts immigrants from the Republic of Cameroon, the Republic of Niger and the Chad Republic $[20,21]$. The diverse inhabitants of Maiduguri predominantly use the Hausa language as the common means of communication and commercial activities [22].

\section{The International Physical Activity Questionnaire}

The questionnaire collects information on the time (i.e., number of days and average time per day) spent being physically active and measures vigorous-intensity activity, moderate-intensity activity, walking activity, and sitting in the last seven consecutive day period. These activity categories may be treated separately to obtain the specific activity patterns or multiplied by their estimated value in Metabolic Equivalent of Tasks (METs) and summed to gain an overall estimate of physical activity in a week http://www.ipaq.ki.se. One MET represents the energy expended while sitting quietly at rest and is equivalent to $3.5 \mathrm{ml} / \mathrm{kg} / \mathrm{min}$ of $\mathrm{VO}_{2}$ Max [23]. The MET intensity values used to score IPAQ questions in this study were vigorous (8 METs), moderate (4 METs) and walking (3.3 METs) http://www.ipaq.ki.se. The IPAQ sitting question is an indicator of the time spent in sedentary activity and was not included as part of the summary score of total physical activity. Data were cleaned to ensure that the daily time spent on each of vigorous, moderate and walking activities ranged between 10 and 180 minutes for all participants http://www.ipaq.ki.se.

The outcomes measures used in the present study were (1) minutes reported in vigorous, moderate, walking and sedentary activities per week (Min week ${ }^{-1}$ ) and (2) MET minutes per week. Time spent in each activity category was derived by multiplying the number of days per week with the minutes spent doing the activity per day, while total weekly physical activity (MET-Min week ${ }^{-1}$ ) was calculated by multiplying the number of minutes spent in each activity category with the specific MET score for each activity.

\section{Procedures}

To establish good face and content validity, the translation and cultural adaptation of the IPAQ-SF was performed in several steps following the guidelines prescribed by the IPAQ core group http://www.ipaq.ki.se. The English version of IPAQ-SF was translated into the Hausa language by two independent translators, both native speakers of Hausa who speak, read and write Hausa as well as speak, read and write English. One of the translators was a Hausa language expert based in the Linguistic Department of the University of Maiduguri, and the second translator was a physiotherapist clinician at a teaching hospital who was knowledgeable about the concept of cardiorespiratory fitness examined in IPAQ. The two translations, one each from the translators, were synthesized into a single Hausa version by a panel of experts consisting of the translators, two physiotherapists who specialize in exercise physiology and sports physiotherapy, the principal investigator, and a university student who was raised in Hausa language and culture. Following back translations of the synthesized Hausa version by two university lecturers from the Hausa ethnic group in Nigeria, each of them with over seven years of professional experience in health sciences, the panel of experts compared the two back translations with 
the original English version to ensure that the concept measured by IPAQ had not been lost during the translations.

The synthesized translation and the two back translations were then merged into one pre-final version of the Hausa IPAQ-Short Form (Hausa IPAQ-SF). The expert panel then compared the original English IPAQ and the pre-final Hausa version for conceptual equivalence (conceptual meaning to terms and concept in the Hausa population), experiential equivalence (cultural relevance of the tasks and examples used in the questionnaire), linguistic equivalence (meaning and grammatical correctness), and metric equivalence (ensuring that the substituting cultural items and examples of activity are equivalent in intensity with the original items and examples). The compendium of physical activity (available at http://prevention.sph.sc. edu) was used by the expert panel to ensure that the substitute culturally acceptable examples and items were equivalent in MET to the original examples and items in the English version.

The pre-final version of the Hausa IPAQ-SF was selfadministered to 12 Hausa-speaking and Hausa-writing volunteers from a broad range of backgrounds. After the completion of the survey, each of the volunteers was separately interviewed by the principal investigator for their understanding of the words in the questionnaire, the clarity of each item, and their opinion and suggestions for improvement. They were also asked to indicate if any question made them feel uncomfortable and if any relevant items were not included in the questionnaire. Items that were difficult to understand as expressed by two or more volunteers were referred to the expert panel for consideration and the panel's recommendations were incorporated in all such cases.

\section{Evaluation of Psychometric Properties}

The final version of the Hausa IPAQ-SF (Additional file 1) was evaluated for validity and reliability in a non-probability sample of 102 apparently healthy individuals, age 20-65 years. Participants for the psychometric study were recruited directly from various workplaces (e.g., university, teaching hospital, private establishments) and neighborhoods in Maiduguri city. Demographic characteristics including educational level and employment status of the participants were obtained on their first day of contact for the study. Height and weight were measured using standardized equipment. Body mass index (BMI) was calculated as body weight divided by the square of height $(\mathrm{kg}$ $\mathrm{m}^{-2}$ ). Participants were eligible for this study if they were willing to complete surveys in both English and Hausa languages and were not having any disability that prevented independent walking. One of the researchers (F.O.O) was in attendance to provide translation and interpretation assistance to participants $(\mathrm{n}=7)$ who were unable to independently complete the survey.

Concurrent validity of the Hausa IPAQ-SF was assessed by comparing the durations (Min week ${ }^{-1}$ ) of physical activity (vigorous, moderate, walking and sitting) from the Hausa IPAQ-SF with that obtained from the original English version of IPAQ-SF. A simple random technique (flip of coin) was used to determine the order of administration of the two questionnaires. Participants whose coin returned with heads completed the Hausa IPAQ-SF first, while those with tails completed the original English version first. An interval of one hour was allowed between administrations of the two questionnaires. Craig et al. [6], in a 12-country international study on validity and reliability of IPAQ, directly compared different versions of IPAQ and described the process as concurrent or inter method validation.

For construct validity, the participants' rate pressure product (RPP) was compared with duration $\left(\right.$ Min week $^{-1}$ ) of physical activity (vigorous, moderate, walking and sitting) from the Hausa IPAQ-SF. Rate pressure product (RPP) is an index of cardiorespiratory fitness and was derived by multiplying participants' resting systolic blood pressure and heart rate [23]. The systolic blood pressure and heart rate were measured with the Dinamap (model $8100 / 8101$ ) digital blood pressure measuring device. Three measurements were taken at intervals of 3-5 minutes, and the mean systolic blood pressure and heart rate were used for computing the RPP used in the analysis. A correlation between duration in physical activity and RPP would suggest that the Hausa IPAQ-SF was sensitive to construct of physical activity such as cardiorespiratory fitness. Other important construct validity measures, such as $\mathrm{VO}_{2}$ max, motion sensors, diaries and indicators of lipid and glucose metabolism [12-14], were not used in this study due to cost and ease of utility.

Test-retest reliability was completed by administering the Hausa IPAQ-SF twice within a 1-week time frame. Participants during the retest administration were asked to focus on the 7-day physical activity recalled during the first testing. The 7-day time interval was chosen because IPAQ asks about activity in the last 7 days. All participants provided informed consent and the Research Ethical Committee of the University of Maiduguri Teaching Hospital, Nigeria gave approval for the protocol of the study before its commencement.

\section{Statistical Analysis}

Descriptive data were reported as mean, standard deviation and percentages. Mean group differences in physical activity (Min week ${ }^{-1}$ ) by gender and socioeconomic status were examined by independent $\mathrm{t}$-test. To assess validity, the non-parametric Spearman correlation coefficients $(\rho)$ 
were calculated to assess the relationship between Min week $^{-1}$ of physical activity from the Hausa IPAQ-SF and rate pressure product and body mass index for construct validity, and with Min week ${ }^{-1}$ in physical activity from the English version of IPAQ for concurrent validity. Pearson correlation coefficients were also used for comparisons, but we only present results from the Spearman correlation analyses, because our results and conclusions were similar. The two-way mixed model (single measure) intraclass correlation coefficient (ICC) was calculated to evaluate the test-retest reliability. A 95\% confidence interval was used to describe the variety/differences in the ICCs by gender and socioeconomic status. Percent agreement was not reported because it overestimated the reliability estimates of our data. To prevent type 2-error due to the small sample size in the socioeconomic statusbased analyses, education and employment status were respectively grouped into two categories: high school education or no high school education, and employed or not employed (homemaker, student, retired, or unable to work). The agreement levels ratings of poor (0-0.2), fair (0.2-0.4), moderate (0.4-0.6), substantial (0.6-0.8) and almost perfect (0.8-1.0), suggested by Landis and Koch, were used when interpreting the reliability results [24].

The Bland-Altman analysis was used to provide an indication of the heteroscedasticity of the data, and $95 \%$ limits of agreement were used for describing the total error between the Hausa IPAQ-SF and the original English IPAQ, and the retest Hausa IPAQ. Variables used for the Bland-Altman analysis were weekly time spent in moderate activity according to the Hausa IPAQ-SF versus the original English IPAQ, and weekly time in moderate activity on first administration of Hausa IPAQ-SF versus the retest administration. Data were entered and analyzed using Statistical Package for the Social Science (SPSS), version 15.0 for windows (SPSS Inc., Chicago, Illinois, USA) and the level of significance was set at $\mathrm{p}<0.05$.

\section{Results}

The discrepancies observed during the translation and cultural adaptation of English IPAQ-SF into Hausa are presented in Table 1. For the test of the pre-final version of Hausa IPAQ-SF, 7 participants out of the 12 surveyed were available for interview after they completed the questionnaire ( 4 men, 3 women; mean age 39.4 years; age range 31-53 years). During the testing of the pre-final version, two items were changed because they raised confusion among the participants. For example, the word 'aikin karfi', an Hausa translation of physical activity, can be misunderstood as referring to being restless, so adding the phrase 'physical exercise' (i.e., motsa jiki) was needed to clarify that it was referring to a health behavior. Walking to travel from place to place was originally translated to 'tafiya daga wannan wuri zuwa wancan', but was frequently understood by almost every participant as walking to travel out of town. It was finally translated to 'tafiya (tattaki) daga wannan wuri zuwa wancan', to indicate walking to move from place to place either for recreation, leisure, exercise, or sports.

The mean age of the subjects who participated in the psychometric testing was $36.2 \pm 9.5$ years (range $=20-65$ years) and the mean body mass index was $23.9 \pm 4.3 \mathrm{~kg} /$ $\mathrm{m}^{2}$ (range $\left.=15.4-35.2 \mathrm{~kg} / \mathrm{m}^{2}\right)$. The participants resting systolic blood pressure, diastolic blood pressure and heart rate were $125.9 \pm 19.4 \mathrm{mmHg}$ (range $=90-217), 81.3 \pm$ $10.4 \mathrm{mmHg}$ (range $=60-117$ ) and $77 \pm 10.1$ beat $\mathrm{min}^{-1}$

\section{Table 1 Examples of discrepancies identified during cross-cultural adaptation of IPAQ and their resolution}

\begin{tabular}{|c|c|}
\hline Issue & Resolution \\
\hline $\begin{array}{l}\text { Selection of vocabulary that responds to the uses and custom in } \\
\text { Hausa to avoid ambiguity on the final Hausa version. }\end{array}$ & $\begin{array}{l}\text { Adjustment of the second person pronoun "you" to third person pronouns "he, } \\
\text { she" to accommodate gender applicability to Hausa version of IPAQ. } \\
\text { In Hausa, the third person pronouns are used for questions because of gender } \\
\text { sensitivity. The words "ka" and "ki" are used respectively to distinguish questions } \\
\text { for male and female. }\end{array}$ \\
\hline $\begin{array}{l}\text { Adjustment of English words to match words with familiar } \\
\text { concept in Hausa/Nigeria. }\end{array}$ & $\begin{array}{l}\text { Vigorous activity: The word "vigorous" can be misunderstood, as it is not } \\
\text { commonly used in Nigeria. The word "very hard" is selected as replacement, } \\
\text { because it is more representative of the language used in Nigeria. } \\
\text { Moderate activity: the word "hard" was used to replace moderate, because the } \\
\text { word is more suitable for the intended activity intensity in Nigeria. }\end{array}$ \\
\hline $\begin{array}{l}\text { Experiential equivalence: selection of activities equivalent to the } \\
\text { Hausa culture. }\end{array}$ & $\begin{array}{l}\text { Item 1: Examples of very hard (vigorous) activities are given that are common in } \\
\text { Hausa cultures/Nigeria such as carrying a heavy bucket of water on the head, } \\
\text { carrying a two-year-old child on the back and farming (making ridges and } \\
\text { hoeing). } \\
\text { Item 2: Hard (moderate) activities commonly engaged in Hausa culture/Nigeria } \\
\text { are provided as examples, such as pounding grains, sweeping compounds and } \\
\text { weeding or planting seedlings. } \\
\text { Item } 3 \text { and } 4 \text { : Walking to the farm and work was added as a common walking } \\
\text { activity in Nigeria. Sitting doing "course work" was replaced with "house work", } \\
\text { and sitting "chatting with families" was included as an example of sedentary } \\
\text { activity in Nigeria. }\end{array}$ \\
\hline
\end{tabular}


(range $=59-109)$, respectively. The majority were men $(\mathrm{n}=56 ; 54.9 \%)$ and married $(\mathrm{n}=69 ; 67.6 \%)$, while $29.4 \%$ $(\mathrm{n}=30)$ and $40.2 \%(\mathrm{n}=41)$ were of the Hausa ethnic group and had university/tertiary education, respectively. A simple majority of the participants were employed $(\mathrm{n}=$ $52 ; 51.0 \%)$. Additional characteristics of the sample are presented in Table 2.

\section{Concurrent validity}

Spearman correlation coefficients ranged from moderate ( $\rho=0.78)$ to high $(\rho=0.92)$, indicating good concurrent validity for the Hausa IPAQ-SF. Total physical activity $\left(\mathrm{MET}\right.$-min week $^{-1}$ ) from the Hausa version of IPAQ-SF was significantly and highly correlated with the total physical activity (MET-min week ${ }^{-1}$ ) from the original English IPAQ-SF $(\rho=0.92, p<0.001)$. High positive and

Table 2 Socio-demographic characteristics of the Participants $(\mathrm{N}=102)$

\begin{tabular}{|c|c|c|}
\hline Characteristics & $n$ & $\%$ \\
\hline \multicolumn{3}{|l|}{ Gender } \\
\hline Men & 56 & 54.9 \\
\hline Women & 46 & 45.1 \\
\hline \multicolumn{3}{|l|}{ Marital Status } \\
\hline Married & 69 & 67.6 \\
\hline Single & 29 & 28.4 \\
\hline Divorced & 4 & 3.9 \\
\hline \multicolumn{3}{|l|}{ Ethnic group } \\
\hline Hausa & 30 & 29.4 \\
\hline Fulani & 11 & 10.8 \\
\hline Shuwa & 9 & 8.8 \\
\hline Kanuri & 19 & 18.6 \\
\hline Others & 33 & 32.4 \\
\hline \multicolumn{3}{|l|}{ Religion } \\
\hline Islam & 81 & 79.4 \\
\hline Christianity & 21 & 20.6 \\
\hline \multicolumn{3}{|l|}{ Educational level } \\
\hline Tertiary & 41 & 40.2 \\
\hline Secondary & 38 & 37.3 \\
\hline Primary & 16 & 15.7 \\
\hline Never attended school & 7 & 6.9 \\
\hline \multicolumn{3}{|l|}{ Employment status } \\
\hline Employed & 52 & 51.0 \\
\hline Retired & 4 & 3.9 \\
\hline Students & 26 & 25.5 \\
\hline Housewife & 9 & 8.8 \\
\hline Unemployed & 11 & 10.8 \\
\hline \multicolumn{3}{|l|}{ Body Mass Index } \\
\hline Underweight & 8 & 7.8 \\
\hline Normal Weight & 59 & 57.8 \\
\hline Overweight & 23 & 22.5 \\
\hline Obese & 12 & 11.8 \\
\hline \multicolumn{3}{|l|}{ Health status } \\
\hline Excellent & 22 & 21.6 \\
\hline Very good & 36 & 35.3 \\
\hline Good & 37 & 36.3 \\
\hline Fair & 7 & 6.9 \\
\hline
\end{tabular}

significant correlations were also found for time spent (Min week ${ }^{-1}$ ) in vigorous $(\rho=0.78, \mathrm{p}<0.001)$, moderate $(\rho=0.86, p<0.001)$ and walking $(\rho=0.83, p<0.001)$ activities between the Hausa IPAQ-SF and the original English version of IPAQ-SF. The time (Min week ${ }^{-1}$ ) spent in sitting from the Hausa IPAQ-SF was also significantly $(\rho=0.89, \mathrm{p}<0.001)$ and positively correlated with the time spent in sitting from the original English version of IPAQ-SF. No meaningful gender and socioeconomic differences were found in the correlation coefficients of all items between the Hausa IPAQ-SF and the original English version (Table 3).

The Bland-Altman plot for the Hausa IPAQ-SF and the English IPAQ demonstrated a small mean difference of $6.19 \mathrm{~min} \mathrm{week}^{-1}$ (not significant). However, the 95\% limits of agreements were wide, ranging from -69.75 to 82.12 min week $^{-1}$. A few participants that reported more than 50 min $^{\text {week }}{ }^{-1}$ of moderate activity were responsible for the wide range of the $95 \%$ limits of agreements (Figure 1).

\section{Construct validity}

Rate Pressure Product (RPP) was only significantly correlated with total time spent in sitting (Min week ${ }^{-1}$ ) from the Hausa IPAQ-SF $(\rho=0.21, p<0.01)$. There was also a weak positive correlation between BMI and time (Min week $\left.^{-1}\right)$ spent in moderate activity $(\rho=0.22, p<0.04)$. Rate pressure product and BMI showed no significant relationships with time (Min week ${ }^{-1}$ ) spent in vigorous and walking activity and total physical activity (MET-min week $^{-1}$ ) from the Hausa IPAQ-SF (table 4). The men reported higher mean time (Min week ${ }^{-1}$ ) in vigorous (93.6 vs 62.6, $\mathrm{p}=0.10$ ), moderate (101 vs $84.6, \mathrm{p}=0.68$ ), walking ( 87.1 vs $54.2, \mathrm{p}=0.01)$ and total physical activity

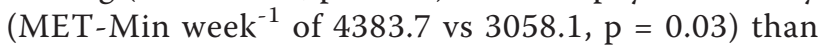
women. However, women spent much more time (Min week $\left.^{-1}\right)$ in sitting activities than men (1773.3 vs 1351.2 ,

Table 3 Concurrent Validity of Hausa IPAQ-SF

\begin{tabular}{|c|c|c|c|}
\hline \multirow[t]{2}{*}{$\begin{array}{l}\text { Hausa IPAQ vs Original } \\
\text { IPAQ }\end{array}$} & $\begin{array}{l}\text { Total } \\
(\mathrm{N}=102)\end{array}$ & $\begin{array}{l}\text { Women } \\
(n=46)\end{array}$ & $\begin{array}{l}\text { Men } \\
(n=56)\end{array}$ \\
\hline & $\rho$ & $\rho$ & $\rho$ \\
\hline Vigorous PA (Min week $^{-1}$ ) & $0.78^{* *}$ & $0.83^{* *}$ & $0.77^{* *}$ \\
\hline Moderate PA (Min week $^{-1}$ ) & $0.86^{* *}$ & $0.82^{* *}$ & $0.90^{* *}$ \\
\hline Walking (Min week ${ }^{-1}$ ) & $0.83^{* *}$ & $0.81^{* *}$ & $0.89^{* *}$ \\
\hline Total PA (MET-Min week ${ }^{-1}$ ) & $0.92^{* *}$ & $0.96^{* *}$ & $0.88^{* *}$ \\
\hline $\begin{array}{l}\text { Time spent sitting (Min } \\
\text { week }^{-1} \text { ) }\end{array}$ & $0.89^{* *}$ & $0.83^{* *}$ & $0.78^{* *}$ \\
\hline $\begin{array}{l}\rho-\text { Spearman correlation coef } \\
\text { from the Hausa IPAQ-SF and } \\
\text { PA = Physical Activity. } \\
\text { Min = Minutes. } \\
\text { MET = Metabolic Energy Turn } \\
* *, p<0.001 .\end{array}$ & $\begin{array}{l}\text { cient betv } \\
\text { e original }\end{array}$ & $\begin{array}{l}\text { e spent } \\
\text { IPAQ. }\end{array}$ & al Activity \\
\hline
\end{tabular}




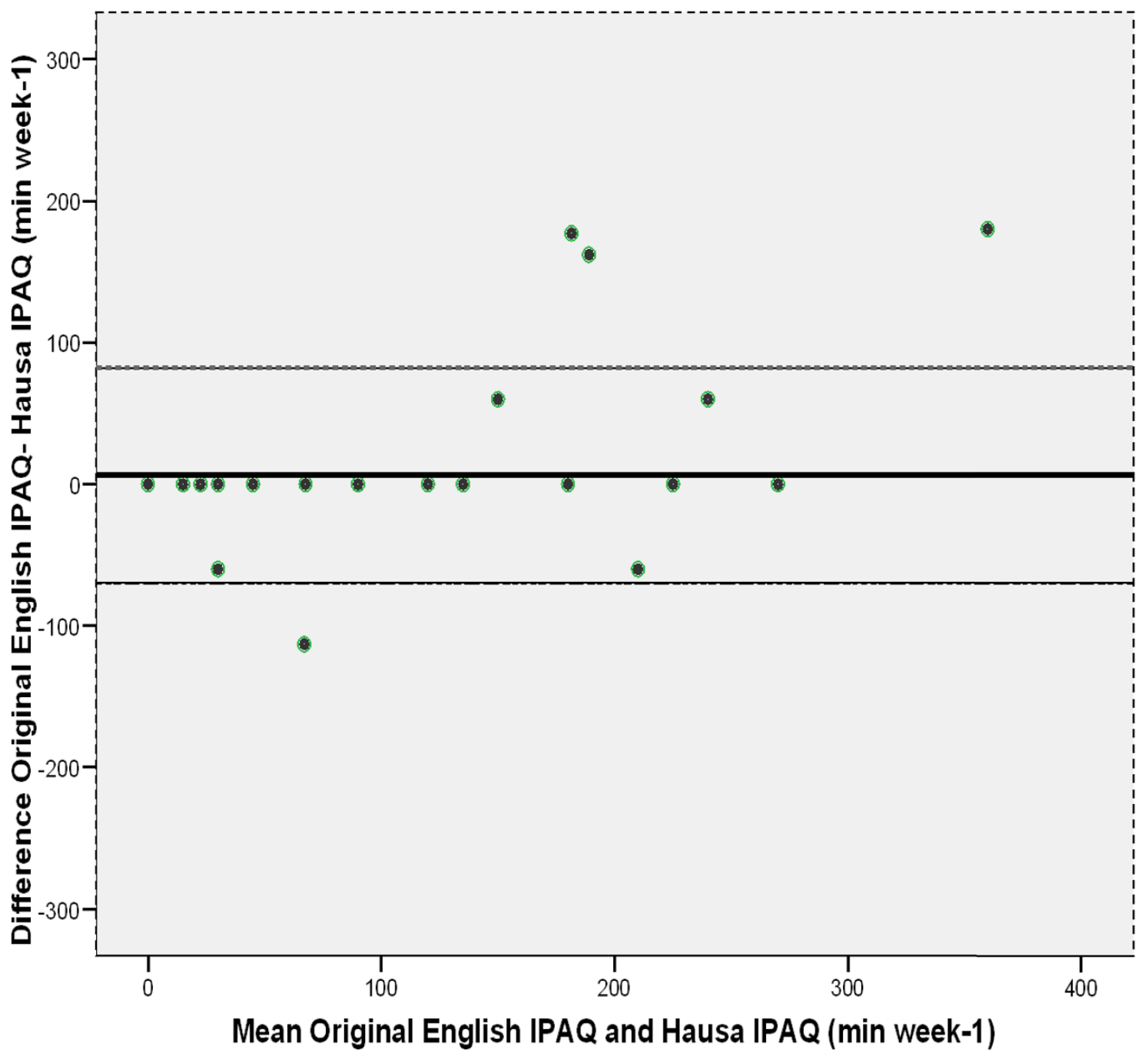

Figure 1 Bland-Altman plot for time spent in at least moderate physical activity (min week-1) as assessed by the English version of International Physical Activity Questionnaire (IPAQ) and measured using the Hausa version of the IPAQ. Mean difference: 6.2 min week1 +/- 2 SD (standard deviations), - 69.8 to 82.1 min week-1 (not significant)

$\mathrm{p}=0.09)$. For educational status, participants that completed high school did not report statistical significant higher mean time (Min week ${ }^{-1}$ ) in doing moderate (98.5 vs $95.8, \mathrm{p}=0.89)$, walking (75.8 vs $66.3, \mathrm{p}=0.56)$ and total physical activity (MET-Min week ${ }^{-1}$ of $3892.3 \mathrm{vs}$ $3417, \mathrm{p}=0.57$ ). Similar those without high school educations did not report statistical significant higher mean time (Min week ${ }^{-1}$ ) in vigorous ( 88.5 vs $81.7, \mathrm{p}=0.77$ ) and sitting (1646.9 vs 1363.3, $\mathrm{p}=0.34$ ) activities. Participants that were employed did not report statistical significant more time (Min week ${ }^{-1}$ ) in vigorous (90.6 vs 72.9, $\mathrm{p}=$ 0.34 ), moderate (107.8 vs $87.8, \mathrm{p}=0.20)$, walking (77.7 vs $70.0, \mathrm{p}=0.56)$ and total physical activity (MET-Min week $^{-1}$ of 4008.5 vs $3571.8, \mathrm{p}=0.57$ ) than those who were unemployed, who reported no statistical significant higher time in sitting activity (1664.1 vs $1504.9, \mathrm{p}=0.52)$ (Not shown in table).

\section{Reliability}

The result of the test-retest reliability is shown in Table 5. Overall, the 1-week ICC ranged from 0.33-0.73, with the lowest value recorded for moderate physical activity and the highest value for vigorous physical activity. The ICC values were significant for all items on the Hausa IPAQ$\mathrm{SF}(\mathrm{p}<0.001)$. Reliability coefficients for vigorous (ICC = $0.82,95 \% \mathrm{CI}=0.67-0.91)$, walking $(\mathrm{ICC}=0.63,95 \% \mathrm{CI}=$ $0.43-0.77)$ and total physical activity ( $\mathrm{CCC}=0.62,95 \%$ $\mathrm{CI}=0.43-0.76)$ were higher in men than in women. The 
Table 4 Construct validity of Hausa IPAQ-SF $(\mathbf{N}=102)$

\begin{tabular}{|c|c|c|}
\hline Hausa IPAQ & Construct measure & $\rho$ \\
\hline Vigorous PA (Min week ${ }^{-1}$ ) & Cardiorespiratory fitness (RPP) & -0.08 \\
\hline Moderate PA (Min week $^{-1}$ ) & Cardiorespiratory fitness (RPP) & -0.09 \\
\hline Walking (Min week ${ }^{-1}$ ) & Cardiorespiratory fitness (RPP) & -0.05 \\
\hline Total PA (MET-Min week $^{-1}$ ) & Cardiorespiratory fitness (RPP) & -0.02 \\
\hline Time spent sitting (Min week ${ }^{-1}$ ) & Cardiorespiratory fitness (RPP) & $0.21 * *$ \\
\hline Vigorous PA (Min week ${ }^{-1}$ ) & $\mathrm{BMI}\left(\mathrm{Kg} \mathrm{m}^{-2}\right)$ & -0.16 \\
\hline$\overline{\text { Moderate PA (Min } \text { week }^{-1} \text { ) }}$ & $\mathrm{BMI}\left(\mathrm{Kg} \mathrm{m}^{-2}\right)$ & $0.22^{*}$ \\
\hline Walking (Min week ${ }^{-1}$ ) & $\mathrm{BMI}\left(\mathrm{Kg} \mathrm{m}^{-2}\right)$ & 0.05 \\
\hline Total PA (MET-Min week $^{-1}$ ) & $\mathrm{BMI}\left(\mathrm{Kg} \mathrm{m}^{-2}\right)$ & 0.18 \\
\hline Time spent sitting (Min week ${ }^{-1}$ ) & $\mathrm{BMI}\left(\mathrm{Kg} \mathrm{m}^{-2}\right)$ & -0.09 \\
\hline
\end{tabular}

$\rho$ _Spearman correlation coefficient between time spent in Physical Activity from the Hausa IPAQ-SF and Cardiorespiratory fitness and BMI.

RPP - Rate Pressure Product, BMI - Body Mass Index.

PA = Physical Activity.

Min = Minutes

MET $=$ Metabolic Energy Turnover.

*, $\mathrm{p}<0.05 ;{ }^{* *}, \mathrm{p}<0.01$.

item demonstrating the highest ICC for women was sitting ( $\mathrm{ICC}=0.67,95 \% \mathrm{CI}=0.48-081)$. Moderate activity resulted in the lowest reliability score for women (ICC = $0.67,95 \% \mathrm{CI}=0.48-0.81$ ).

Table 6 shows the results of the socioeconomic status differences in test-retest reliability of the Hausa IPAQSF. Intraclass Correlation Coefficients for vigorous (ICC $=0.75,95 \% \mathrm{CI}=0.56-0.86)$, moderate $(\mathrm{ICC}=0.36,95 \%$ $\mathrm{CI}=0.13-0.55)$, walking $(\mathrm{ICC}=0.57,95 \% \mathrm{CI}=0.38-$ $0.71)$ and total physical activity $(\mathrm{ICC}=0.62,95 \% \mathrm{CI}=$ 0.46-0.74) were higher among participants with high school educations than in those without high school educations. The reliability coefficient was, however, higher among participants with no high school educations $(\mathrm{ICC}=0.64,95 \% \mathrm{CI}=0.31-0.83)$ than in those with high school educations $(\mathrm{ICC}=0.53$; $95 \% \mathrm{CI}=$ 0.29-0.70) for sitting activity. While ICC was higher among participants that were employed for vigorous activity ( $\mathrm{ICC}=0.73,95 \% \mathrm{CI}=0.49-0.87$ ) and total physical activity $(\mathrm{ICC}=0.65,95 \% \mathrm{CI}=0.45-0.78)$, it was higher for moderate $(\mathrm{ICC}=0.40,95 \% \mathrm{CI}=0.11-0.63$ ), walking ( $\mathrm{ICC}=0.60,95 \% \mathrm{CI}=0.36-0.76)$ and sitting (ICC $=0.58,95 \% \mathrm{CI}=0.36-0.74)$ activities among participants that were unemployed.

A Bland-Altman plot for the test-retest of the Hausa IPAQ-SF showed that the mean differences between time spent in moderate activity ( $\min$ week- ${ }^{1}$ ) on first and second administrations were small $\left(4.33\right.$ min week- $\left.{ }^{1}\right)$ and not significant. However, about ten outliers affected the 95\% limits of agreements and the methods were similar (Figure 2).

\section{Discussion}

This study translated and cross-culturally adapted the English IPAQ-SF, and examined aspects of the validity and reliability of the Hausa-IPAQ-SF in Nigeria. The results indicated that Hausa IPAQ-SF had acceptable metric properties for assessing physical activity in healthy adults.

Participants in the pretesting of the Hausa IPAQ-SF misunderstood the question on "walking to travel from place to place" to mean walking to travel out of town rather than for recreation and for transportation to services and destinations as implied on the original English IPAQ-SF. Misunderstanding of the transportation domain item may be attributed to the distinct infrastructural pattern and transportation habit of the African population. Unlike in many countries in North America, Australia and Europe where leisure time physical activity and recreational walking are prevalent [9,25-27], walking is not traditionally associated with recreation in African societies. Active walking may even be erroneously perceived as a sign of being socioeconomically disadvantaged among the urban African elites.

We found higher concurrent validity of the Hausa IPAQ-SF than that reported in a study that directly compared the long and short versions of IPAQ in one language in 12 countries ( $\rho=0.83-0.92$ vs $\rho=0.51-0.64$ ) [6]. Perhaps a better concurrent validity was found in the present study because, unlike in the 12-country study,

Table 5 Test-retest reliability based on intra-class correlation coefficient for the Hausa IPAQ-SF (N = 102)

\begin{tabular}{|c|c|c|c|}
\hline \multirow[t]{2}{*}{ Hausa IPAQ-SF } & Total $(\mathrm{N}=102)$ & Women $(n=46)$ & Men $(n=56)$ \\
\hline & ICC (95\% Cl) & ICC (95\% Cl) & ICC (95\%CI) \\
\hline Vigorous PA (Min week ${ }^{-1}$ ) & $0.73(0.55-0.84)$ & $0.44(-0.19-0.81)$ & $0.82(0.67-0.91)$ \\
\hline Moderate PA (Min week ${ }^{-1}$ ) & $0.33(0.12-0.51)$ & $0.35(0.05-0.59)$ & $0.31(0.01-0.56)$ \\
\hline Walking (Min week ${ }^{-1}$ ) & $0.56(0.39-0.69)$ & $0.43(0.10-0.67)$ & $0.63(0.43-0.71)$ \\
\hline Total PA (MET-Min week ${ }^{-1}$ ) & $0.61(0.47-0.72)$ & $0.56(0.33-0.73)$ & $0.62(0.43-0.76)$ \\
\hline Sitting (Min week ${ }^{-1}$ ) & $0.56(0.43-0.68)$ & $0.67(0.48-0.81)$ & $0.40(0.15-0.60)$ \\
\hline
\end{tabular}

ICC = Single measure intra-class correlation coefficient.

PA = Physical Activity

Min = Minutes

MET = Metabolic Energy Turnover 
Table 6 Socioeconomic status differences in test-retest reliability of the Hausa IPAQ-SF $(\mathbf{N}=102)$

\begin{tabular}{llllll}
\hline Socioeconomic Status & Vigorous PA & Moderate PA & Walking activity & Total PA & Sitting activity \\
\hline Educational Qualification & & & & & \\
High School $(\mathrm{n}=79)$ & $0.75(0.56-0.86)$ & $0.36(0.13-0.55)$ & $0.57(0.38-0.71)$ & $0.62(0.46-0.74)$ & $0.52(0.34-0.67)$ \\
No High School $(\mathrm{n}=23)$ & $0.67(-0.07-0.93)$ & $0.23(-0.24-0.61)$ & $0.52(0.06-0.79)$ & $0.57(0.21-0.79)$ & $0.64(0.31-0.83)$ \\
\hline Employment Category & & & & & \\
Employed (50) & $0.73(0.49-0.87)$ & $0.25(-0.06-0.52)$ & $0.51(0.24-0.71)$ & $0.65(0.45-0.78)$ & $0.53(0.29-0.70)$ \\
Unemployed (52) & $0.72(0.39-0.89)$ & $0.40(0.11-0.63)$ & $0.60(0.36-0.76)$ & $0.56(0.34-0.72)$ & $0.58(0.36-0.74)$ \\
\hline
\end{tabular}

PA = Physical Activity.

different languages of only the short IPAQ were compared. However, consistent with previous studies where comparisons of IPAQ data were made with accelerometer monitors $[15,28,29]$, we found higher correlation value for the Hausa IPAQ-SF with vigorous intensity activity than with moderate activity and walking. This finding therefore affirms an earlier report that shows the
IPAQ-SF to be more sensitive to intense physical activity than moderate and low intensity activities [12].

In the absence of objective criterion standards for evaluating an absolute estimate of physical activity, the consistency of items on IPAQ with variables known to be related to physical activity such as body mass index (BMI), blood pressure, heart rate, indicators of lipid and

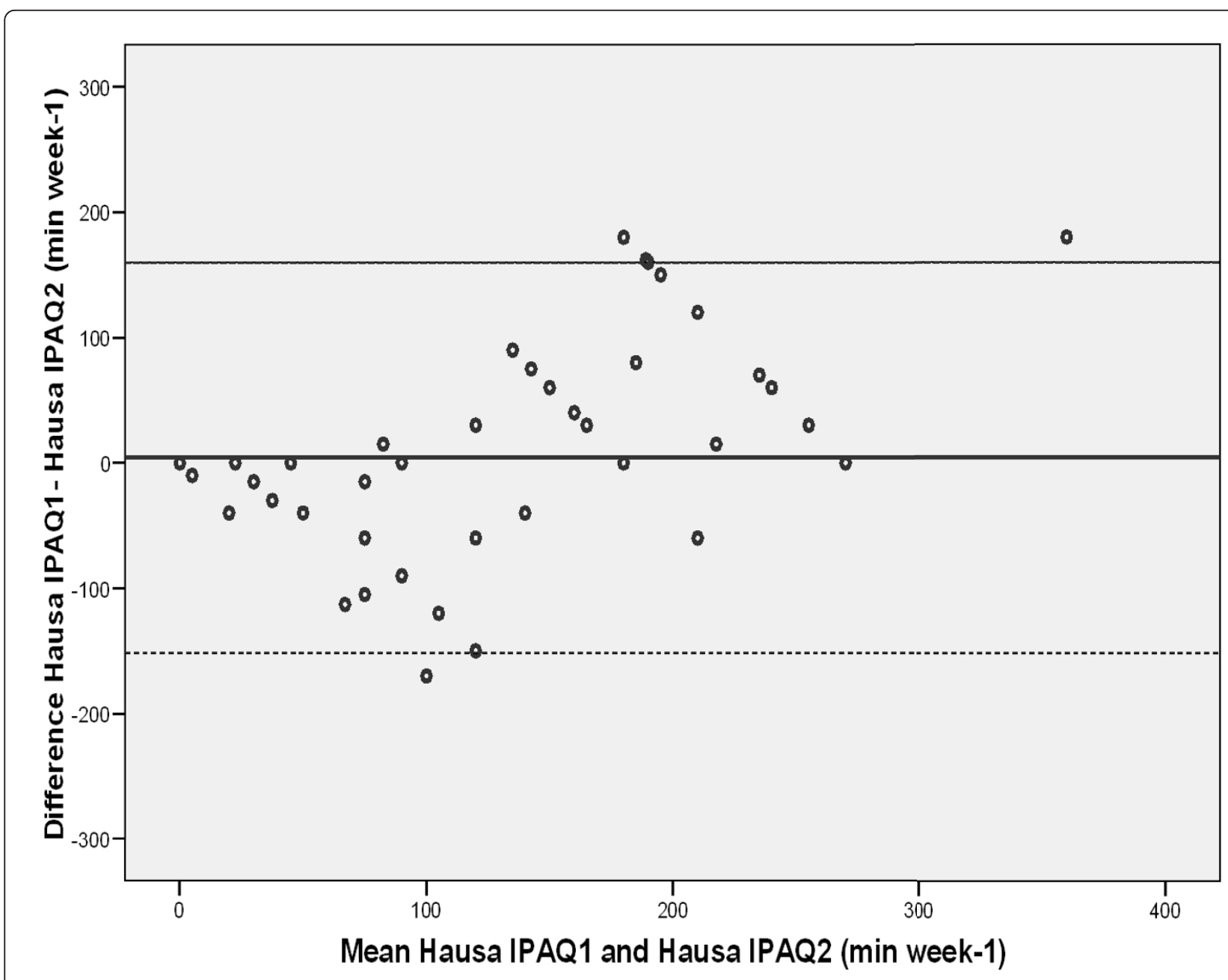

Figure 2 Bland-Altman plot for moderate activity (min week-1) as assessed by Hausa version of the International Physical Activity (IPAQ) on day 1 and after seven days (test retest reliability). Mean difference: 4.3 min week-1 +/- 2 SD (standard deviations), -151.4 to 160.0 min week-1 (not significant) 
glucose metabolism, and fitness index have been used as important validation characteristics $[12,13,28]$, referred to as indirect or construct validity $[13,28,30]$. Poor construct validity of the Hausa IPAQ-SF in the present study is indicated by a weak, albeit significant, correlation only between rate pressure product as an index of cardiorespiratory fitness index and sitting time (Min week $\left.^{-1}\right)(\rho=0.21)$. Significant but tenuous correlation between time spent in sitting and rate pressure product implies that higher sitting time as measured on the Hausa IPAQ-SF is related to poor cardiorespiratory fitness (higher RPP indicates lower cardiorespiratory fitness).

The poor relationship between cardiorespiratory fitness and total physical activity, vigorous activity, moderate activity and walking is inconsistent with those of previous studies that reported weak but significant correlations between indices of cardiorespiratory fitness and time spent in vigorous-intensity activity $[12,13,31,32]$, moderate-intensity activity [28] and total physical activity $[12,13,28]$. Perhaps the contrasting finding between the present study and the previous ones is attributable to the disparate measures of cardiorespiratory fitness utilized. In the present study, RPP as the index of cardiorespiratory fitness incorporated resting heart rate and blood pressure unlike others that utilized submaximal heart rate derived from response to work load as the index of cardiorespiratory fitness $[12,13,28]$. It has been documented that heart rate response to submaximal workload estimates cardiorespiratory fitness or $\mathrm{VO}_{2}$ max better than resting heart rate [23].

The reproducibility of the Hausa IPAQ-SF evidenced by substantial agreement for vigorous $(\mathrm{ICC}=0.73)$ and total physical activity $(\mathrm{ICC}=0.61)$, moderate agreement for walking $(\mathrm{ICC}=0.56)$, and sitting activities $(\mathrm{ICC}=0.56)$ and by fair agreement for moderate activity $(\mathrm{ICC}=0.33)$ is similar to those of other studies [12,33]. A study on a Nordic population reported substantial to almost perfect correlations for vigorous activity and sitting, but similar fair correlation ( $\mathrm{ICC}=0.34$ ) for moderate activity on IPAQ-SF [12]. Another study that assessed the reliability of four physical activity measures including the IPAQ short form in an Australian population found ICC for the questions on moderate activities to be the lowest, ranging from poor to fair agreement (0.16-0.44) [33]. Papathanasiou et al. [34], in a population of Greek young adults found weaker ICC (0.76) for moderate physical activity compared to walking ( ICC $=0.78)$, vigorous $(\mathrm{ICC}=0.88$ ) and total physical activity $(\mathrm{ICC}=0.92)$ on the Greek version of IPAQ-SF.

Relatively low reliability for moderate intensity activities compared to vigorous intensity activities as found in the present study may be explained by the low salience nature of moderate activities as previously hypothesized
[35]. It is plausible that the time spent in moderate physical activities may be incidental and not easily remembered by participants in the present study. Structured vigorously physical activities like sports and exercise can be more easily recalled compared to some vaguely defined moderate physical activity like "carrying light load" or "multiple household tasks at once" as indicated on the IPAQ-SF [34]. Overall good to acceptable reliability coefficients for all items on the Hausa IPAQ-SF indicate that the instrument is reproducible and internally consistent and is comparable to the findings in previous studies [12,33,34].

Our finding of meaningfully higher reliability coefficient for vigorous intensity activity among men than women is not surprising, because the participants who are men also reported spending more time in all physical activity categories except in sedentary time than the women. Consistently, men have been found to be more physically active than women in a Nigerian population [36]. It is therefore plausible for men in Nigeria to be more consistent in response to items that pertained to intense physical activity than to sedentary activity. Similarly, no statistical significance differences were found in the socioeconomic-based analyses, but participants with higher socioeconomic status as indicated by education (high school education) and employment (those employed) were more physically active than those with low socioeconomic status, and more consistent in their response to questions on vigorous and total physical activity. While it may be difficult to draw any definite conclusion from these findings, they reflect the potential influence of gender and socioeconomic status on physical activity assessment.

Direct comparison of our findings with previous studies should be made with caution, because in the majority of these studies, the accelerometer was utilized as a common objective criterion standard to validate the IPAQ $[14,15,28]$. In low-income developing countries, the availability and cost of accelerometers remain important research issues [6], and poor receptivity to wearing accelerometers for physical activity assessment has been reported in a sample of urban African adults [5]. Furthermore, the divergent reports on construct validity may suggest that a single measure of aerobic capacity may not be sufficient to determine the construct validity of the physical activity questionnaire. In order to prevent spurious validity of physical activity questionnaires, the selection of comparison instruments that can accurately assess the construct or related dimension measured by the questionnaire has been advocated [37].

Non-utilization of a physical activity diary or any objective measure as a criterion standard to evaluate the validity of the Hausa IPAQ-SF is one limitation of the present study. The choice and availability of appropriate 
criterion measures are particular issues of concern for the validation of physical activity questionnaires in the low-income countries of Africa [5,38]. In addition, the generalizability of this study may be limited due to the convenience sampling technique utilized and the relative low sample size. Also, the majority of participants were government employees and students with potential higher comprehension and recall ability than may be found in the general population. However, recruitment from diverse workplaces and neighborhoods allowed for a sample with reasonable heterogeneity in age, educational level and ethnic backgrounds.

Despite these limitations, the study demonstrated the feasibility of using the Hausa IPAQ-SF to reliably collect physical activity data in a diverse segment of the Nigerian population. This is the first study in an African country to explore the cultural adaptation and translation of the IPAQ, warranting the need for further validation studies of the Hausa version of IPAQ-SF in other African populations. However, because some of the participants in the present study required assistance to complete the survey, participants in any future national studies in Africa would need to be asked about their ability to independently complete the Hausa IPAQ-SF.

\section{Conclusion}

In conclusion, our results indicate that the Hausa IPAQSF has acceptable concurrent validity and test-retest reliability for assessing vigorous intensity, walking, sitting and total physical activity in Nigerian adults. Moderate intensity activity demonstrated almost perfect concurrent validity, but only fair test-retest reliability. The construct validity of Hausa IPAQ-SF provided poor agreement with the rate pressure product and with the BMI, reflecting the need for further assessment in a large sample with objective physical activity and fitness measures, such as the accelerometer and $\mathrm{VO}_{2} \mathrm{Max}$.

\section{Additional material}

Additional file 1: Hausa Version of IPAQ-SF. A final version of the Hausa translated IPAQ-SF and back translated English copy.

\footnotetext{
Acknowledgements

The authors wish to thank Messrs. A Shuiabu, A Rislanu, and A Jajere and Mrs. M.B Saleh for their technical assistance with the IPAQ translation and back translation process. We would also like to thank all the participants that took part in this study. Funding

None

\section{Author details}

${ }^{1}$ Department of Physiotherapy, College of Medical Sciences, University of Maiduguri, Nigeria. ${ }^{2}$ Department of Physiotherapy, College of Medicine, University of Ibadan, Nigeria.
}

\section{Authors' contributions}

ALO conceived, designed and provided coordination for the study: conducted the statistical analysis, interpreted the data, and drafted the manuscript. AYO contributed to study design and helped to draft the manuscript. BOA and FOO participated in the design of the study, data acquisition and drafting of the manuscript. HNA, SUA and AAR all contributed to critically revising the drafted manuscript for important intellectual content. All authors read and approved the final manuscript.

\section{Competing interests}

The authors declare that they have no competing interests.

Received: 18 January 2011 Accepted: 22 November 2011 Published: 22 November 2011

\section{References}

1. World Health Organization: Reducing risks, promoting healthy life. World Health Report 2002 Geneva: World Health Organization; 2002.

2. Abegunde DO, Mathers CD, Adam T, Ortegon M, Strong K: The burden and costs of chronic diseases in low-income and middle-income countries. Lancet 2007, 370:1929-1938.

3. World Health Organization: Reducing risks, promoting healthy life World Health Report 2002. Geneva: World Health Organization; 2002.

4. World Health Organization: Preventing chronic diseases: a vital investment Geneva, Switzerland: World Health Organization; 2005.

5. Sobnqwi E, Mbanya JCN, Urwin NC, Aspray TJ, Alberti KGMM: Development and validation of a questionnaire for the assessment of physical activity in epidemiological studies in Sub-saharan Africa. Int J Epidemiol 2001, 30:1361-1368.

6. Craig $\mathrm{CL}$, Marshall AL, Sjostrom M, Bauman AE, Booth ML, Ainsworth BE et al: International physical activity questionnaire: 12 country reliability and validity. Med Sci Sports Exerc 2003, 35(8):1381-1395.

7. The International Physical Activity Questionnaire. Short Last 7 Days SelfAdministered Format 2002 [http://www.ipaq.ki.se], Accessed March 21, 2011

8. Bauman A, Bull FC, Craig CL, Chey T, Ainsworth BE, Sallis JF, et al: The International prevalence study on physical activity: results from 20 countries. Int J Behav Nutr Phys Act 2009, 6:21 [http://www.ijbnpa.org/ content/pdf/1479-5868-6-21.pdf].

9. Sallis JF, Bowles HR, Bauman, Ainsworth BE, Bull FC, Craig CL, et al: Neighborhood environments and physical activity among adults in 11 countries. Am J Prev Med 2009, 36:484-490.

10. Guthold R, Ono T, Strong KL, Chatterji S, Morabia A: Worldwide variability in physical inactivity: a 51-country survey. Am J Prev Med 2008, 34:486-494.

11. Maddison R, Mhurchu CN, Jiang Y, Hoorn SV, Rodgers A, Lawes CMM, Rush E: International physical activity questionnaire (IPAQ) and New Zealand physical activity questionnaire (NZPAP): A doubly labeled water validation. Int J Behav Nutr Phys Act 2007, 4:62.

12. Kurtze N, Rangul V, Hustveldt B: Reliability and validity of the international physical activity questionnaire in the Nord-Trondelag Health Study (HUNT) population of men. BMC Med Res Methodol 2008, 8:63.

13. Graff-Iversen S, Anderssen SA, Holme IM, Jenum AK, Raastad T: An adapted version of the long international physical activity questionnaire (IPAQ L): Construct validity in a low income, multiethnic population study from Oslo, Norway. Int J Behav Nutr Phys Act 2007, 4:13.

14. Ekelund U, Sepp H, Brage S, Becker W, Jakes R, Hennings M, Wareham N: Criterion-related validity of the last 7-day, short form of the international physical activity questionnaire in Swedish adults. Public Health Nutr 2006, 2:258-265.

15. Qu NN, Li KJ: Study on the reliability and validity of international physical activity questionnaire (Chinese Vision, IPAQ). Zhonghua Liu Xing Bing Xue Za Zhi 2004, 25:265-268, (Abstract).

16. Oyeyemi AL, Adegoke BOA, Oyeyemi AY, Fatudimu MB: Test-retest reliability of IPAQ environmental-module in an African population. Int $J$ Behav Nutr Phys Act 2008, 5:38.

17. Mulero-Portela AL, Colon-Santaella CL, Cruz-Gomez C: Cross-cultural adaptation of the disability of arm, shoulder, and hand questionnaire: Spanish for Puerto Rico Version. Int J Rehabil Res 2009, 32(4):287-293.

18. National African Language Resources Center: Hausa language and culture National African Language Resources Center (NALC): University of Wisconsin-Madison, USA; 2010 
19. Wikipedia. Maiduguri [http://en.wikipedia.org/wiki/Maiduguri], Accessed (November 27, 2010)

20. National Bureau of Statistics: Statistical fact sheet and population census Federal Republic of Nigeria; 2008 [http://www.nigerianstat.gov.ng], Accessed (January 25, 2011).

21. Kagu MB, Ahmed SG, Mohammed AS, Moshood WK, Malah MB, Kehinde JM: Anti-A and Anti-B Heamolysins among group "O" voluntary blood donors in Northeastern Nigeria. Journal of Transfussion 2011 [http:// www.hindawi.com/journals/itf/2011/302406/], Accessed (November 17. 2011), ID 302406.

22. National Population Commission (NPC) [Nigeria] and ORC Macro: Nigeria Demographics and Health Survey 2003 Calverton, Maryland: National Population Commission and ORC Macro; 2004.

23. Pollock ML, Willmore JH: In Exercise in health and disease: Evaluation and prescription for prevention and rehabilitation. 2 edition. Edited by: Pollock ML, Willmore JH. WB Saunders Company, Philadelphia; 1990:89-230.

24. Landis J, Koch G: The measurement of observer agreement for categorical data. Biometrics 1977, 33:159-174.

25. Humpel N, Owen N, Leslie E, Marshal AL, Bauman AE, Sallis JF: Associations of location and perceived environmental attributes with walking in Neighborhoods. Am J Health Prom 2004, 18(3):239-242.

26. Owen N, Humpel N, Leslie E, Bauman A, Sallis JF: Understanding environmental influences on walking: review and research agenda. Am J Prev Med 2004, 27:67-76

27. De Boudeaudhuij I, Texeira PJ, Cardon G, Deforche B: Environmental and psychosocial correlates of physical activity in Portuguese and Belgian Adults. Public Health Nutr 2005, 8(7):886-895.

28. Hagstromer M, Oja P, Sjostrom M: The international physical activity questionnaire (IPAQ) a study of concurrent and construct validity. Public Health Nutr 2006, 9:755-762.

29. Prince SA, Adamo KB, Hamel ME, Hardt J, Gorber SC, Tremblay M: A comparison of direct versus self-report measures for assessing physical activity in adults: a systematic review. Int I Behav Nutr Phys Act 2008, 5:56.

30. Washburn RA, Heath GW, Jackson AW: Reliability and validity issues concerning large scale surveillance of physical activity. Res Q Exerc Sport 2000, 71:104-13.

31. Perreira MA, FitzerGerald SJ, Gregg EW, Joswiak ML, Ryan WJ, Suminski RR, et al: A collection of physical activity questionnaire for health-related research [special issue]. Med Sci Sports Exerc 1997, 29:S1-205.

32. Wareham NJ, Jakes RW, Rennie KL, Mitchel J, Hennings S, Day NE: Validity and repeatability of the EPIC-Norfolk physical activity questionnaire. Int $J$ Epidemiol 2002, 31:168-174.

33. Brown WJ, Trost SG, Bauman A, Mummery K, Owen N: Test-retest reliability of four physical activity measures used in population surveys. Journal of Science and Medicine in Sport 2004, 7:205-215.

34. Papathanasiou G, Georgoudis G, Papandreou M, Spyropoulous P, Georgakopoulos D, Kalfakakou V, Evangelou A: Reliability measures of the short international physical activity questionnaire (IPAQ) in Greek young adults. Hellenic Journal of Cardiology 2009, 50:283-294.

35. Sallis JF, Saelens BT: Assessment of physical activity by self-reports: Status, limitations and future directions. Res Q Exerc Sport 2000, 71:1-14.

36. Adegoke BOA, Oyeyemi AL: Physical inactivity in Nigerian young adults: prevalence and sociodemographic correlates. J Phys Activity Health 2011, 8:1135-1142.

37. Wareham N, Rennie K: The assessment of physical activity in individuals and populations: why try to be more precise about how physical activity is assessed. Int $J$ Obes 1999, 22(suppl 2):S30-S38.

38. Wareham NJ: Commentary: Measuring physical activity in sub-saharan African. Int J Epidemiol 2001, 30:1369-1370.

\section{Pre-publication history}

The pre-publication history for this paper can be accessed here: http://www.biomedcentral.com/1471-2288/11/156/prepub

\section{doi:10.1186/1471-2288-11-156}

Cite this article as: Oyeyemi et al.: The short international physical activity questionnaire: cross-cultural adaptation, validation and reliability of the Hausa language version in Nigeria. BMC Medical Research Methodology 2011 11:156.

\section{Submit your next manuscript to BioMed Central and take full advantage of:}

- Convenient online submission

- Thorough peer review

- No space constraints or color figure charges

- Immediate publication on acceptance

- Inclusion in PubMed, CAS, Scopus and Google Scholar

- Research which is freely available for redistribution

Submit your manuscript at www.biomedcentral.com/submit
Ciomed Central 